\title{
Contribution des réserves profondes du sol au bilan hydrique des cultures. Détermination et importance
}

\author{
Nader KATERJI, François DAUDET $\left({ }^{*}\right)$ \& Charles VALANCOGNE $\left({ }^{* *}\right)$ \\ avec la collaboration technique de Maurice BACH \& Alain FOUĖRE \\ I.N.R.A., Station de Bioclimatologie, Route de Saint-Cyr, F 78000 Versailles \\ (*) I.N.R.A., Station de Bioclimatologie, Domaine de Crouelle, F 63000 Clermont Ferrand \\ (**) I.N.R.A., Station de Bioclimatologie, Domaine Duclos, FWI 97170 Petit Bourg
}

RÉSUMÉ

En associant aux mesures de variations du stock d'eau du sol $\Delta Q$ et de pluie $P$, un suivi continu de l'évapotranspiration réelle ETR (par la méthode du rapport de BOWEN), il est possible d'estimer les flux profonds D de drainage ou de remontée d'eau en appliquant l'équation du bilan hydrique :

$$
\mathrm{D}=\mathrm{P}-\Delta \mathbf{Q}-\mathrm{ETR} \text {. }
$$

La méthode a été appliquée pendant 4 années sur blé d'hiver (Triticum aestivum) et luzerne (Medicago sativa) cultivés sur sol limoneux profond. Les résultats (fig. 1, 2, 3, 4 et tabl. 5) indiquent que l'importance des flux observés à $170 \mathrm{~cm}$ de profondeur varie essentiellement en fonction de la climatologie de l'année considérée. En période pluvieuse, le ruissellement peut conduire à une mauvaise estimation du flux de drainage. En période sèche, d'importantes quantités d'eau $(100 \mathrm{~mm})$ proviennent des couches plus profondes que $170 \mathrm{~cm}$, aussi bien pour le blé que pour la luzerne. Différents mécanismes pouvant expliquer ces remontées d'eau sont envisagés. Par ailleurs, le mode d'utilisation de l'eau du sol par ces 2 cultures est comparé.

Mots clés additionnels : Drainage, remontée en eau, blé, luzerne.

By associating measurements of variation in soil water reserves $\Delta Q$ and rainfall $P$, with continuous measures of actual evapotranspiration ETR (using the BowEN ratio method), it was possible to estimate the deep water rise or drainage fluxes $\mathrm{D}$ by applying the water balance equation :

$$
\mathrm{D}=\mathrm{P}-\Delta \mathrm{Q}-\mathrm{ETR} \text {. }
$$

This method was applied for 4 years on winter wheat (Triticum aestivum) and lucerne (Medicago sativa) grown in deep silt soil. The results (fig. 1, 2, 3,4 and table 5) showed that the magnitude of the fluxes observed at a depth of $170 \mathrm{~cm}$ varied essentially according to the climatology of the year in question. During a rainy period, runoff could lead to a poor estimation of drainage flow. During a dry period, large quantities of water $(100 \mathrm{~mm})$ arrived from layers below $170 \mathrm{~cm}$, for wheat as well as for lucerne. Various mechanisms which could account for these water rises are considered. And a comparison is made of the way in which soil water is used by the two crops.

Additional key words : Drainage, water rise, wheat, lucerne.

\section{INTRODUCTION}

La consommation d'eau d'une culture par évapotranspiration peut être évaluée en effectuant le « bilan hydrique " du sol, méthode qui tend à chiffrer les quantités d'eau qui entrent, sortent ou demeurent dans un volume spécifique de sol au cours d'une période de temps donnée. Les différents termes du bilan hydrique sont reliés par l'équation suivante :

$$
\mathrm{P}+\mathrm{I}-\Delta \mathrm{Q}-\mathrm{ETR}-\mathrm{D} \pm \mathrm{R}=0
$$

avec :

$\Delta \mathrm{Q}$ : variation du stock d'eau dans le sol pour un intervalle de temps donné et pour une 
tranche de sol $[\Delta \mathrm{Z}]_{0}^{Z}$ comprise entre la surface et une profondeur $z$ de référence.

P, I, ETR : respectivement les pluies, l'irrigation et l'évapotranspiration réelle cumulées pour le même intervalle de temps.

D: flux vertical d'eau à la profondeur $z$, exprimé ici positivement pour un drainage, négativement en cas de remontée d'eau.

$\pm \mathrm{R}$ : apport ou perte latérale d'eau par ruissellement superficiel.

L'équation (1) fait abstraction de flux latéraux divergents pouvant exister dans la masse du sol.

La mesure directe de l'ETR restant relativement difficile, cette composante du bilan hydrique est assez mal connue malgré son importance, tant du point de vue agronomique qu'hydrologique. Le plus souvent en effet, l'ETR est déduite de l'équation simplifiée du bilan hydrique suivante :

$$
\mathrm{ETR}=\mathrm{P}-\Delta \mathrm{Q} .
$$

Comparée à la relation générale (1), l'équation (2) revient à admettre implicitement que les flux profonds (D) ainsi que les apports par ruissellement $( \pm R$ ) sont nuls. S'il est parfaitement légitime dans de nombreuses situations et en considérant les périodes peu pluvieuses de négliger le terme de ruissellement superficiel, de nombreux travaux suggèrent que les quantités d'eau susceptibles de transiter dans un sens ou dans l'autre entre la zone racinaire et le sous-sol représentent une composante importante du bilan hydrique (ROBINS et al., 1954 ; NIXON \& LAWLESS, 1960 ; WILCOX, 1960 ; ROSE \& STERN, 1965 ; La RUE et al., 1968 ; VAN BAVEL et al., 1968b ; CARVALLoet al., 1975). Or, ces flux profonds intéressent l'agronomie non seulement sous l'angle du bilan hydrique ou de l'efficience de l'eau, mais aussi sous celui du bilan minéral puisqu'ils s'accompagnent de mouvement de divers solutés impli- qués dans l'équilibre de fertilité des sols. Ainsi, les phénomènes de salinisation des couches supérieures du sol ou la pollution des nappes profondes sont-ils des conséquences de ces mouvements.

L'étude des flux profonds est possible, en principe, par la méthode du bilan hydrique si le ruissellement est négligeable, à condition de disposer d'une mesure directe de l'ETR et de la variation du stock d'eau du sol $\Delta Q$. Le flux profond est donné alors par :

$$
\mathrm{D}=\mathrm{P}-\Delta \mathrm{Q}-\mathrm{ETR} \text {. }
$$

L'objet de cet article est de présenter des résultats de mesure de flux profonds de drainage ou de remontée d'eau à partir de cette équation. Les observations ont porté sur 4 années de pluviosité assez variable et concernant 2 types de culture : une graminée, le blé (Triticum aestivum) et une légumineuse, la luzerne (Medicago sativa).

\section{MATÉRIEL ET MÉTHODES}

\section{A. Les cultures et les conditions de milieu}

L'étude a été réalisée au domaine expérimental de La Minière en région parisienne. Les cultures envisagées ont été successivement un blé d'hiver (variété « Talent ») en 1975 et 1976 et une luzerne (variété « du Puits » semée en 1977) en 1978 et 1979.

La pluie et l'évapotranspiration potentielle (formule de Penman) mensuelles sont données dans les tableaux 1 et 2 pour les années considérées. Notons que la pluviosité sur le site de La Minière est généralement bien répartie au cours de l'année avec une moyenne mensuelle de $50 \mathrm{~mm}$ (soit $1,7 \mathrm{~mm} /$ jour). Les années étudiées présentent des écarts marqués par rapport à cette

TABLEAU 1

Précipitations mensuelles et annuelles au cours des 4 années étudiées ( $\mathrm{mm}$ ).

\begin{tabular}{|c|c|c|c|c|c|c|c|c|c|c|c|c|c|}
\hline Année Mois & Janv. & Févr. & Mars & Avr. & Mai & Juin & Juill. & Août & Sept. & Oct. & Nov. & Déc. & Total \\
\hline 1975 & 66 & 17 & 69 & 39 & 78 & 45 & 109 & 35 & 128 & 24 & 71 & 13 & 694 \\
\hline 1976 & 13 & 34 & 26 & 19 & 10 & 1 & 68 & 2 & 47 & 33 & 47 & 64 & 364 \\
\hline 1978 & 73 & 83 & 102 & 64 & 58 & 62 & 42 & 40 & 49 & 8 & 9 & 115 & 705 \\
\hline 1979 & 28 & 71 & 104 & 66 & 55 & 32 & 24 & 65 & 16 & 60 & 60 & 104 & 685 \\
\hline
\end{tabular}
Monthly and annual rainfall ( $\mathrm{mm}$ ) during the 4 years under study.

TABLEAU 2

Evapotranspirations mensuelles et annuelles (formule de PENMAN) au cours des 4 années étudiées (mm).

\begin{tabular}{|c|c|c|c|c|c|c|c|c|c|c|c|c|c|}
\hline Année Mois & Janv. & Févr. & Mars & Avr. & Mai & Juin & Juill. & Août & Sept. & Oct. & Nov. & Déc. & Total \\
\hline 1975 & 24 & 24 & 38 & 77 & 102 & 120 & 129 & 113 & 72 & 36 & 19 & 11 & 765 \\
\hline 1976 & 19 & 25 & 52 & 97 & 110 & 148 & 146 & 134 & 68 & 44 & 16 & 12 & 871 \\
\hline 1978 & 16 & 25 & 48 & 64 & 87 & 110 & 108 & 104 & 63 & 33 & 10 & 15 & 683 \\
\hline 1979 & 12 & 18 & 40 & 72 & 111 & 116 & 127 & 98 & 69 & 32 & 13 & 15 & 723 \\
\hline
\end{tabular}
Monthly and annual potential evapotranspiration (PENMAN formula), during the 4 years under study (mm). 
situation moyenne. Ainsi, en 1975 et 1978 , la pluviosité a été relativement abondante (respectivement 2,04 et $2,08 \mathrm{~mm} / \mathrm{j}$ ), alors qu'en 1979 , elle était nettement inférieure à la normale $(1,14 \mathrm{~mm} / \mathrm{j})$. L'année 1976 , quant à elle, est caractérisée par une sécheresse exceptionnelle avec une pluviométrie annuelle inférieure à $1 / 3$ de la normale $(0,56 \mathrm{~mm} / \mathrm{j})$.

L'étude pédologique détaillée du Domaine a été réalisée par JONGERIUS (1954). La parcelle est constituée de sols profonds limoneux en situation de plateau. Ces sols sont décrits comme des limons tachetés devenant calcaires en profondeur. La composition granulométrique de la couche $0-30 \mathrm{~cm}$ est donnée tableau 3 . Les profils de densité apparente (méthode des cylindres) et des humidités volumiques correspondant à la capacité au champ observée in situ sont donnés tableau 4 . Nous avons considéré comme "capacité au champ " l'humidité du sol mesurée de façon très stable par la méthode neutronique en février et début mars 1976 après reconstitution des réserves hydriques du sol et en l'absence de drainage sensible. Dans cet état, le contenu total en eau de la tranche $0-170 \mathrm{~cm}$ était de $590 \mathrm{~mm}$.

\section{TABLEAU 3}

Composition granulométrique de l'horizon $0-30 \mathrm{~cm}$ (\% pondéral). Mechanical composition of the top $30 \mathrm{~cm}$ layer of the soil.

\begin{tabular}{cccc}
\hline \hline $\begin{array}{c}\text { Argile } \\
(<0,002 \mathrm{~mm})\end{array}$ & $\begin{array}{c}\text { Limon } \\
(0,002 \mathrm{~mm}-0,02 \mathrm{~mm})\end{array}$ & $\begin{array}{c}\text { Sable fin } \\
(0,02 \mathrm{~mm}-0,2 \mathrm{~mm})\end{array}$ & $\begin{array}{c}\text { Sable grossier } \\
(>0,2 \mathrm{~mm})\end{array}$ \\
\hline 10,6 & 78,0 & 8,4 & 3,0 \\
\hline
\end{tabular}

TABLEAU 4

Profil de densité apparente (méthode des cylindres) et de teneur volumique en eau à la capacité au champ (méthode neutronique, février 1976) sur une des parcelles étudiées.

Apparent density (undisturbed core method) and volume water content at field capacity (neutron probe, February 1976) in one plot.

\begin{tabular}{lccccccc}
\hline $\begin{array}{l}\text { Profondeur } \\
(\mathrm{cm})\end{array}$ & 20 & 40 & 70 & 100 & 130 & 160 \\
\hline $\begin{array}{l}\text { Densité } \\
\text { apparente }\end{array}$ & 1,32 & 1,38 & 1,49 & 1,52 & 1,55 & 1,60 \\
\hline $\begin{array}{l}\text { Capacité } \\
\text { au champ }\end{array}$ & 0,330 & 0,333 & 0,354 & 0,348 & 0,342 & 0,354 \\
\hline \hline
\end{tabular}

L'humidité pondérale au pF 4,2 déterminée au laboratoire pour la couche $0-30 \mathrm{~cm}$ est de $8 \mathrm{p} .100$, ce qui conduirait à une humidité volumique de l'ordre de 11 p. 100 en admettant une densité apparente de 1,37 (au lieu de 1,32, compte tenu d'une légère rétraction du sol à l'état sec). La « réserve utile », telle qu'on la définit classiquement, est donc de l'ordre de $(0,33-0,11)$ $\times 300=66 \mathrm{~mm}$ pour la couche $0-30 \mathrm{~cm}$. Cette valeur serait toutefois à nuancer, compte tenu des mesures de potentiel hydrique effectuées par VALANCOGNE \& DAUDET (1977) sur le même sol : la teneur en eau à pF 4,2 in situ est systématiquement inférieure à celle observée en laboratoire sur le même sol remanié.

\section{B. Mesure du stock d'eau du sol et de ses variations.}

La variation du stock d'eau dans le sol a été déterminée à l'aide d'un humidimètre à neutrons (HOLMES, 1950) dont l'étalonnage selon la méthode de COUCHAT (1974) a été contrôlé à diverses reprises par comparaison avec des mesures d'humidité pondérale (VALANCOGNE et al., 1977). Les mesures neutroniques sont effectuées à une cadence décadaire, notamment pendant la période estivale. Le stock d'eau du sol est obtenu en cumulant les valeurs d'humidité mesurées de 10 en $10 \mathrm{~cm}$, entre 15 et $165 \mathrm{~cm}$ de profondeur. La mesure à $15 \mathrm{~cm}$ est supposée représentative de la tranche de sol $0-20 \mathrm{~cm}$. Selon les années, 4 ou 5 tubes d'accès en alliage d'aluminium ont été installés dans une aire d'étude limitée, d'environ $20 \mathrm{~m}^{2}$, située au centre de la parcelle et à proximité du dispositif de mesure de l'ETR.

L'analyse statistique des mesures obtenues par la méthode neutronique a fait l'objet de plusieurs études (NIELSEN et al., 1973 ; VAUCLIN et al., 1982 ; VACHAUD et al., 1983). Il en ressort que la variabilité naturelle du stock d'eau du sol entre les différents points de mesure est très importante et conduit à une assez faible précision sur la valeur absolue de la quantité d'eau présente à un instant donné. Par contre, les valeurs de variation du stock d'eau au cours du temps obtenues par cette méthode sont assez précises. DAUDET \& VALANCOGNE (1976) ont montré sur le site de La Minière qu'avec une moyenne calculée sur 5 emplacements (tubes d'accès), l'intervalle de confiance obtenu sur la variation du stock d'eau du sol est de l'ordre de $5 \mathrm{~mm}$ pour un profil de $170 \mathrm{~cm}$ de profondeur.

\section{Mesure de l'évapotranspiration réelle}

Diverses techniques ont été mises en œuvre successivement pour approcher l'ETR sur le site de La Minière. Les cuves pesables (GREBET, 1965 ; PERRIER et al., 1974), si elles ont fourni les premières valeurs précises de l'ETR maximale de diverses cultures, ne permettent pas d'apprécier sur de longues périodes l'ETR de cultures non irriguées, car l'existence d'un fond étanche qui interdit les échanges d'eau avec le sous-sol, les rend surtout en période sèche - peu représentatives du champ avoisinant (KATERJI et al., 1977). Cette remarque établit l'intérêt des méthodes suivantes qui s'appuient sur des mesures météorologiques et/ou aérodynamiques pour calculer l'ETR :

- la méthode du rapport de Bowen (SLATYER \& Mc ILROY, 1961), dont une des réalisations est le système BEARN (PERRIER et al., 1975, 1976) où l'ETR est calculée à partir de mesures du rayonnement net (bilanmètre), du flux de chaleur dans le sol (fluxmètre) et de la température de thermomètres secs et mouillés (psychromètres) à 2 niveaux au-dessus de la culture,

- la méthode aérodynamique (LEMON, 1968), qui fait appel au rayonnement net ainsi qu'aux profils de vitesse du vent et de température au-dessus de la végétation,

- la méthode aérodynamique combinée (GRANT, 1975), d'où dérive le système SAMER (ITIER, 1981), dans laquelle interviennent les mesures du rayonnement net et celles de température et de vitesse du vent à 2 niveaux. 
Il n'entre pas dans le propos de cet article de discuter de l'intérêt comparé de ces diverses méthodes dont l'emploi en continu a été rendu possible par les progrès récents en matière de mesure et de traitement de l'information. L'étude présente a été réalisée à l'aide du système BEARN. Des comparaisons systématiques des données fournies par ce système avec celles obtenues par un évapotranspiromètre pesable placé dans les mêmes conditions ont été effectuées pendant plusieurs années (PERRIER et al., 1976 ; KATERJI, 1977 ; KATERJI et al., 1977). Ces confrontations ont montré que les valeurs cumulées de l'ETR fournies par les 2 méthodes sont très proches, les différences observées étant de l'ordre de 5 p. 100 en moyenne. Nous admettrons que l'erreur relative sur l'ETR cumulée des diverses cultures que nous avons étudiées est de cet ordre.

La pluviométrie $\mathrm{P}$ a été mesurée quotidiennement à proximité immédiate de la parcelle d'essai.

\section{RÉSULTATS EXPÉRIMENTAUX}

\section{A. Les flux profonds et leur importance dans le bilan hydrique des cultures étudiées}

Les figures 1, 2, 3, 4 présentent les résultats obtenus de 1975 à 1979. Les grandeurs suivantes ont été repré-

$$
\begin{aligned}
& \text { Orainage } \\
& \left(\mathrm{mm} . \mathrm{j}^{-1}\right)
\end{aligned}
$$
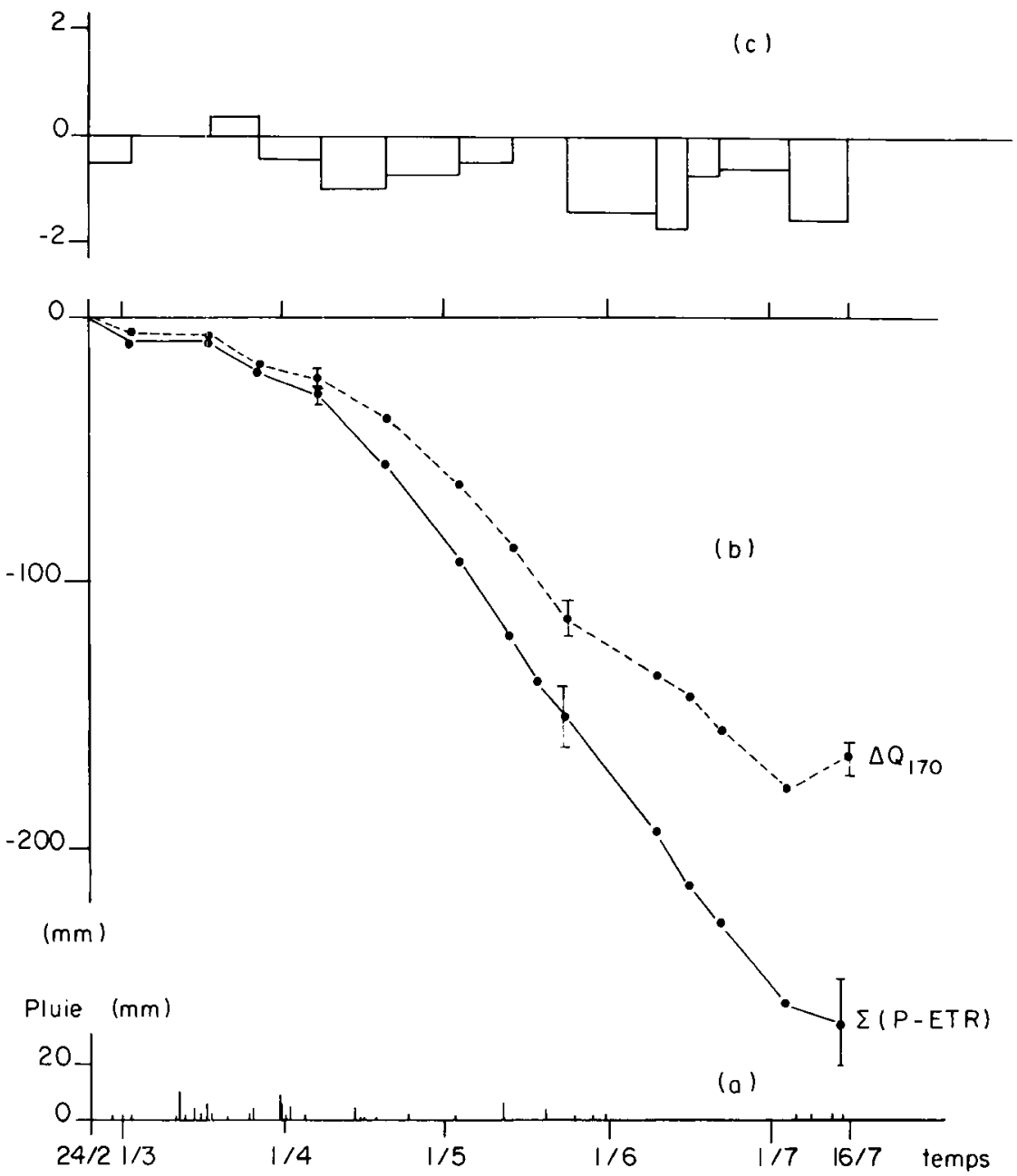

$\left(\mathrm{mm} \mathrm{j}^{-1}\right)$
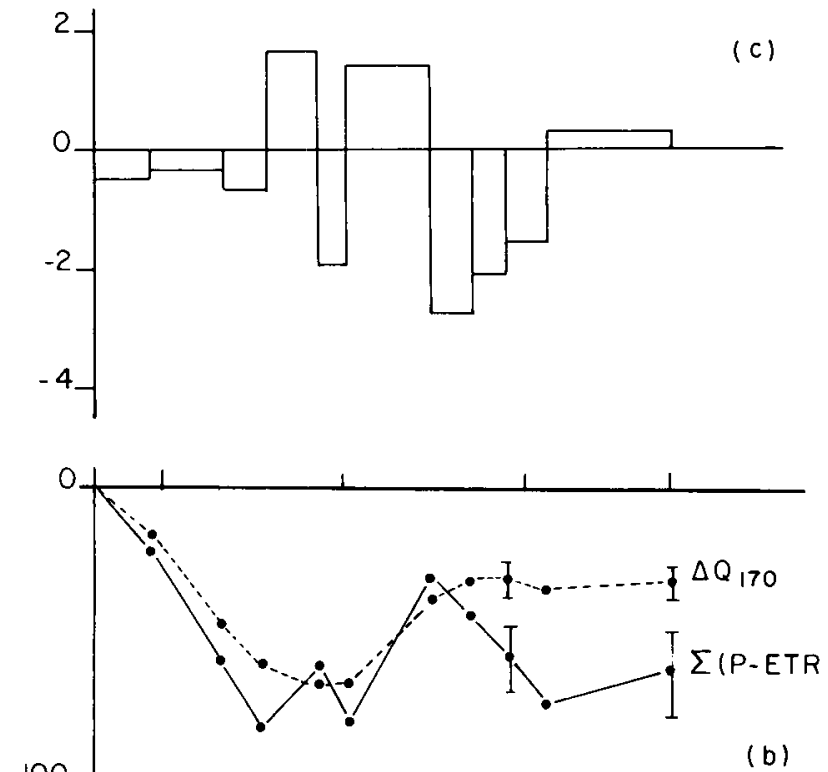

$(\mathrm{mm})$

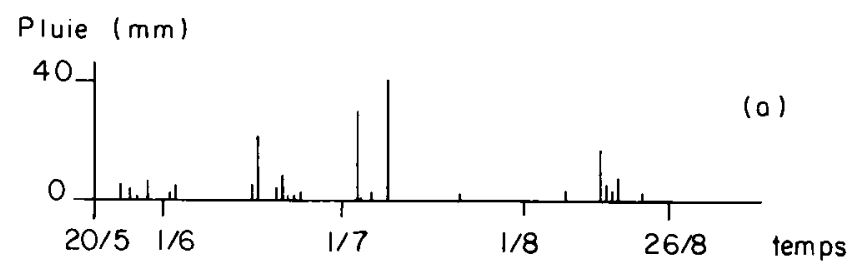

\section{Figure 1}

Evolution des différents termes du bilan hydrique d'une culture de blé d'hiver pendant l'année 1975 .

a) Précipitations.

b) $\Delta Q_{170}$ : écart du contenu total en eau dans la tranche $0-170 \mathrm{~cm}$ de sol par rapport à la date origine.

$\Sigma(P-E T R):$ différence cumulée entre pluviométrie et évapotranspiration réelle depuis la date origine.

c) Drainage moyen à $170 \mathrm{~cm}$ de profondeur (compté positivement vers le bas).

Water balance components for a winter wheat crop during the year 1975 .

a) Rainfall.

b) $\Delta Q_{I 70}$ : variation of the total water content in the 0-170 cm upper layer of the soil since an initial date.

$\Sigma(P-E T R):$ cumulative difference between the rainfall and the actual evapotranspiration since the same initial date.

c) Average flux of draingge at the depth $170 \mathrm{~cm}$ (positive downward).

Figure 2

Evolution des différents termes du bilan hydrique d'une culture de blé d'hiver pendant l'année 1976.

$a, b, c$, même signification que pour la figure 1 . Water balance components for a winter wheat crop during the year 1976.

$a, b, c$, same as in figure $I$. 
Drainage

$\left(\mathrm{mm} . \mathrm{j}^{-1}\right)$


Figure 3

Evolution des différents termes du bilan hydrique d'une culture de luzerne pendant l'année 1978.

$a, b, c$, même signification que pour la figure $l$.

sentées sur la base des périodes de temps imposées par la méthode neutronique :

1) Etat du stock d'eau $Q$ contenu dans la tranche de sol $0-170 \mathrm{~cm}$, par rapport à une date origine correspondant à la capacité au champ (absence de drainage apparent). Le contenu total en eau du sol sur $170 \mathrm{~cm}$ de profondeur au moment considéré, comme la capacité au champ, est relativement stable d'une année à l'autre et égal à $600 \pm 30 \mathrm{~mm}$.

2) Valeur cumulée de $\Sigma(\mathrm{P}-\mathrm{ETR})(\mathrm{mm})$ à partir de la même date origine. L'écart entre ces 2 courbes provient des termes non contrôlés du bilan hydrique (flux profonds, cf. équation 3).
Water balance components for a lucerne crop during the year 1978. $a, b, c$, same as in figure 1 .

3) Valeur moyenne pour chaque période $\Delta t$ de mesure du flux profond $\frac{\Sigma(\mathrm{P}-\mathrm{ETR})-\Delta \mathrm{Q}}{\Delta \mathrm{t}}(\mathrm{mm} / \mathrm{jour})$. Une valeur positive correspond à une perte d'eau pour la tranche de sol $0-170 \mathrm{~cm}$. Compte tenu du mode de représentation adopté, la surface des rectangles est proportionnelle à la quantité totale $(\mathrm{mm})$ mise en jeu par ce terme du bilan hydrique, au cours de la période considérée.

4) Pluies journalières.

Par ailleurs, les valeurs cumulées des différents termes du bilan hydrique pour les 4 saisons d'observation sont présentées dans le tableau 2. 

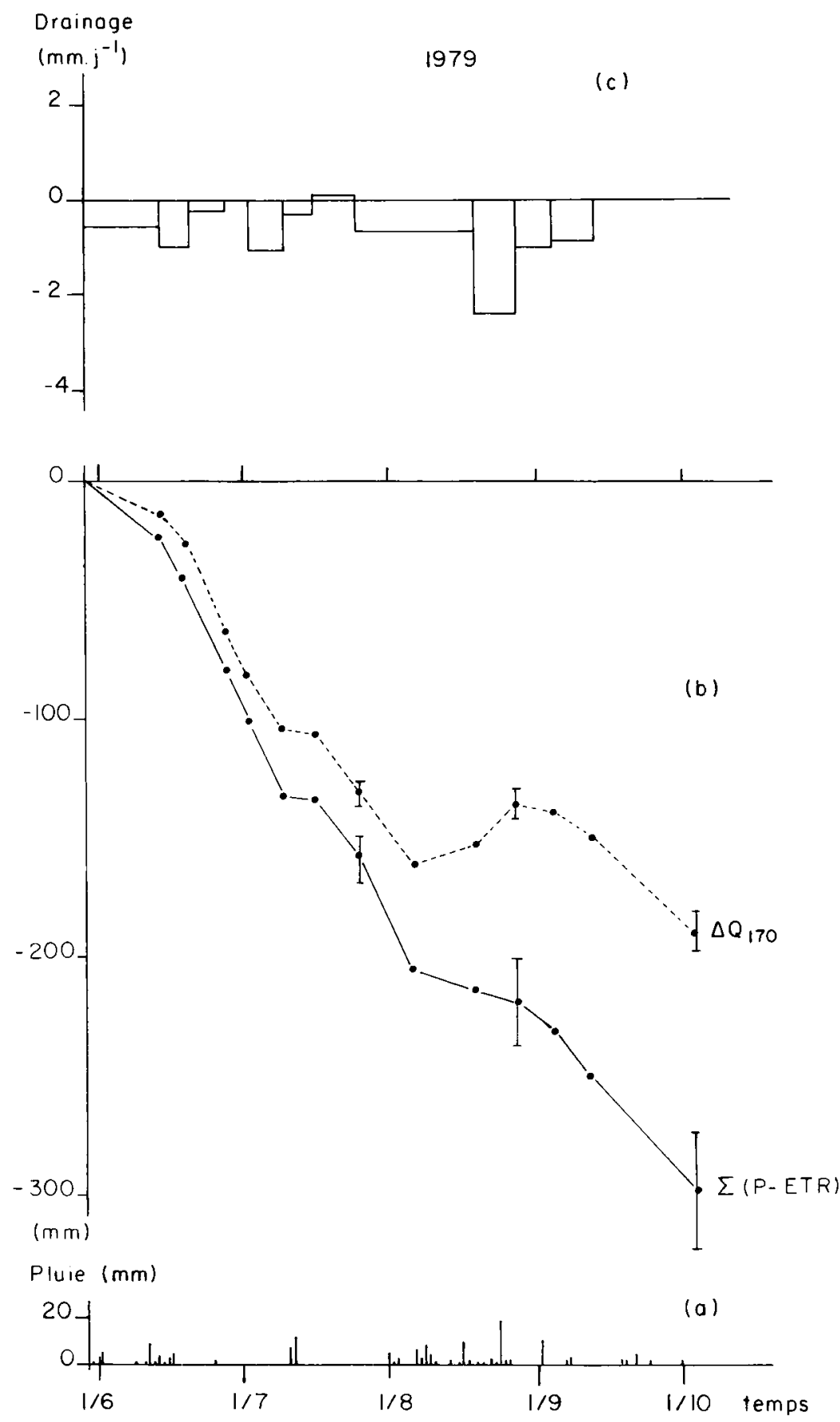

Figure 4

Evolution des différents termes du bilan hydrique d'une culture de luzerne pendant l'année 1979.

$a, b, c$, même signification que pour la figure 1 .

Water balance components for a lucerne crop during the year 1979. $a, b, c$, same as in figure $I$.

Ces différents résultats conduisent aux remarques suivantes :

- Les flux profonds existent à différentes périodes, que ce soit en année sèche ou humide, et représentent un élément non négligeable du bilan hydrique.

- Les années humides (1975 et 1978, fig. 1 et 3) présentent globalement un équilibre entre les quantités d'eau perdues par drainage et récupérées par remontée, puisqu'en fin de période, le déficit hydrique réel calculé $\Sigma(P-E T R)$ est voisin de la variation observée du stock d'eau $\Delta Q$ (cf. également tabl. 5). Cependant, le drainage profond peut représenter une composante importante du bilan hydrique, notamment pendant la période humide du printemps 1978 (fig. 3).

- Pour les années à été sec (1976 et 1979, fig. 2 et 4), la courbe $\Sigma(P-E T R)$ évolue dans un premier temps parallèlement à la courbe $\Delta \mathrm{Q}$. Ceci correspond à des périodes de pluviométrie faible mais régulière. Les 2 courbes se séparent ensuite de plus en plus nettement en période sèche. A la fin de la période d'observation, le déficit réel calculé $\Sigma(\mathrm{P}-\mathrm{ETR})$ est supérieur à la variation de stock d'eau du sol constatée dans la tranche $0-170 \mathrm{~cm}$, traduisant pour ces 2 années, une remontée totale d'eau d'environ $100 \mathrm{~mm}$, aussi bien pour le blé que pour la luzerne. 


\section{TABLEAU 5}

Résultats globaux des bilans hydriques et de leurs composantes pendant les périodes d'observation.

$P=$ Pluviométrie, $E T R=$ Evapotranspiration, $\Delta Q_{170}=$ variation du stock d'eau contenu dans la tranche de sol $0-170 \mathrm{~cm}$, $D_{I 70}=$ drainage à $170 \mathrm{~cm}$ de profondeur (positif vers le bas).

Results on the overall water balance and its different components during the periods of observation. $P=$ Rainfall, ETR = actual evapotranspiration, $A Q_{170}=$ variation of the total water content in the 0-170 cm upper layer of the soil, $D_{170}=$ drainage at the depth $170 \mathrm{~cm}$ (positive downward).

\begin{tabular}{|c|c|c|c|c|c|c|c|}
\hline \multirow{2}{*}{ Année } & \multirow{2}{*}{ Culture } & \multirow{2}{*}{ Période } & \multicolumn{5}{|c|}{ Composantes cumulées du bilan hydrique (mm) } \\
\hline & & & $\mathbf{P}$ & ETR & $(P-$ ETR $)$ & $\Delta \mathrm{Q}_{170}$ & $\mathrm{D}_{170}$ \\
\hline 1975 & Blé d'hiver & $20 / 05-26 / 08$ & 204 & 285 & -.81 & -66 & -15 \\
\hline 1976 & Blé d'hiver & $21 / 02-16 / 07$ & 76 & 342 & -266 & -166 & -100 \\
\hline 1978 & Luzerne & $08 / 03-13 / 09$ & 394 & 536 & -142 & -162 & +20 \\
\hline 1979 & Luzerne & $29 / 05-03 / 10$ & 144 & 435 & -291 & -191 & -100 \\
\hline
\end{tabular}

\section{B. Les modalités du prélèvement d'eau dans la tranche 0-170 cm.}

Sur les 4 années étudiées, les profils de déficit par rapport à la capacité au champ, obtenus à différentes dates et au moment où le sol présente le stock d'eau minimum, sont présentés figure 5 . On constate que :

- sous-blé, l'essentiel des variations de stock d'eau sur la tranche $0-170 \mathrm{~cm}$ a lieu dans le $1^{\mathrm{er}} \mathrm{m}$ de sol. Ceci est observé aussi bien en année sèche (1976) qu'en année humide (1975).

- sous luzerne, la variation du stock d'eau est mieux répartie en profondeur bien que la contribution de la couche supérieure reste la plus importante.

- En examinant les profils des déficits maximums observés en juillet 1976 sous blé et juillet 1978 sous luzerne, on constate des différences marquées entre les

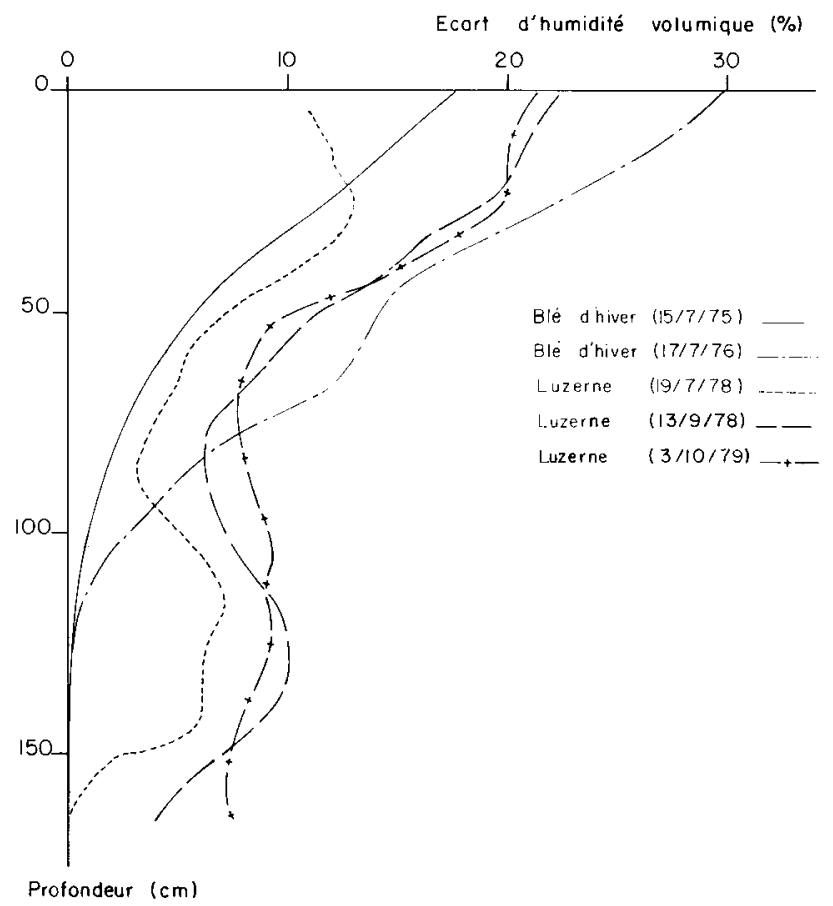

Figure 5

Ecarts d'humidité volumique maximums observés par rapport à la capacité au champ, lors des périodes les plus sèches.

Maximum difference of the volumetric water content with respect to field capacity for the driest periods.
2 espèces : le blé semble mieux utiliser le stock d'eau dans la couche supérieure, par contre la luzerne profite davantage du stock d'eau des couches profondes du sol.

\section{DISCUSSION ET CONCLUSION}

Les résultats présentés dans les figures $1,2,3$ et 4 ainsi que dans le tableau 2 indiquent clairement que les flux profonds du sol ne sont pas des composantes marginales du bilan hydrique et qu'ils doivent par conséquent être pris en considération.

En année humide et notamment après des pluies orageuses, une quantité d'eau non négligeable (environ $50 \mathrm{~mm}$, fig. 3) est perdue. Notons que, malgré la très faible pente des terrains considérés, certaines pluies particulièrement violentes peuvent occasionner des phénomènes de ruissellement. Il devient alors difficile d'affirmer qu'au cours de telles périodes orageuses, cette perte d'eau s'explique par le seul drainage profond. Le ruissellement intervient pour une part que l'on peut supposer d'autant plus importante que la culture est moins couvrante (cas précisément de la luzerne en mars 1978, fig. 3), mais nos investigations actuelles ne nous permettent pas de préciser, dans ce cas, le poids relatif du ruissellement et du drainage profond.

Les remontées d'eau en année sèche sont importantes, de l'ordre de $100 \mathrm{~mm}$ pour les 2 cas étudiés : 1976, où elles représentent environ 30 p. 100 de l'ETR mesurée sur blé, et 1979 , où cette quantité représente 23 p. 100 de l'ETR mesurée sur la luzerne. Il faut donc admettre que les besoins en eau des cultures peuvent être couverts à partir des réserves présentes dans le sol, y compris dans des couches très profondes (profondeur $>2 \mathrm{~m}$ ) considérées le plus souvent comme peu susceptibles de contribuer de manière significative à la satisfaction de ces besoins. Les observations que nous avons rapportées semblent indiquer qu'une mobilisation importante des réserves profondes du sol est possible en période de sécheresse marquée. Notons que, malgré la sécheresse exceptionnelle de l'année 1976, les sols profonds, du type de ceux étudiés ici, ont conduit à une récolte céréalière parfaitement satisfaisante (KATERJI, 1977), ce qui tendrait à confirmer l'importance du phénomène évoqué.

Pour la luzerne, les remontées d'eau qui apparaissent 
à $170 \mathrm{~cm}$ de profondeur sont très probablement liées essentiellement à une absorption intense par les racines de la plante dans les horizons plus profonds (cf. fig. 5). Cette particularité de la luzerne a déjà été soulignée par LiPPS \& FOX (1964) ou par MAERTENS \& CABELGUENNE (1974) qui signalent par ailleurs que l'intensité de l'absorption semble peu en rapport avec une biomasse racinaire qui décroît très fortemeri en profondeur. Des prélèvements effectués sur nos parcelles montrent qu'environ 80 p. 100 de la masse racinaire occupent la tranche $0-30 \mathrm{~cm}$ pour la luzerne et la tranche 0 $75 \mathrm{~cm}$ pour le blé; dans les 2 cas, une fraction non négligeable atteint des profondeurs supérieures à $100 \mathrm{~cm}$.

Pour le blé, il est frappant de constater que, malgré l'importance des flux décelés, aucune baisse sensible de l'humidité du sol n'apparaît en dessous de $120 \mathrm{~cm}$ de profondeur. On peut dans ces conditions essayer d'expliquer les remontées d'eau par 2 phénomènes :

- Phénomène de remontée capillaire: la présence d'un impurtant réservoir d'eau en profondeur (nappe ou horizons voisins de la saturation sur une épaisseur relativement grande) conduirait à un écoulement de caractère quasi stationnaire à travers les couches situées en dessous de $120 \mathrm{~cm}$, vers les horizons supérieurs du sol soumis au dessèchement. Le dessèchement du sol en étant réparti sur des épaisseurs importantes pourrait devenir inférieur à la limite de sensibilité de l'humidimètre à neutrons, donc non détectable.

On sait que la seule mesure des variations de teneur en eau du sol est, même si l'on utilise des tubes très longs, insuffisante pour déterminer le régime et la direction de l'écoulement (VAN BAVEL, 1968 $a$ et $b$ ), et que, même dans le cas où la teneur en eau reste constante à une profondeur donnée, cela ne signifie pas l'absence de flux à cette profondeur puisqu'un régime permanent est possible (HILLEL, 1974). Des mesures tensiométriques peuvent cependant préciser l'intensité et la direction des gradients de charge hydraulique qui sont associés aux flux d'eau du sol. Il devient alors possible, théoriquement, de calculer ces flux si l'on connaît par ailleurs la conductivité hydraulique du sol et sa variation en fonction de la teneur en eau (PHILIP, 1957 ; GARDNER, 1958). Les essais dans ce sens effectués par DAUDET \& VALANCOGNE (1976) sur le sol étudié ici (mais à $85 \mathrm{~cm}$ de profondeur) ont montré que des remontées capillaires de l'ordre de $1 \mathrm{~mm} /$ jour sont plausibles lorsqu'on se situe au voisinage de la capacité au champ, compte tenu de la conductivité capillaire et des gradients de potentiel hydrique mesurés.
- Absorption racinaire dans des couches de sol plus profondes que $170 \mathrm{~cm}$ en 1976 : il est possible que les racines du blé aient exploré des zones de sol beaucoup plus profondes qu'en année normale ; on pourrait alors imaginer que malgré une absorption apparemment très faible entre 120 et $170 \mathrm{~cm}$, des horizons plus profonds et, peut-être, plus densément prospectés aient été le siège d'une activité racinaire relativement intense. Ce type de raisonnement trouve des appuis dans un certain nombre de travaux qui soulignent l'importante extension verticale et horizontale des systèmes racinaires chez le blé ainsi que chez de nombreuses graminées. Certains auteurs cités par BALDY (1973) (KMOCHCTAL, 1957 ; KAZKHOU \& GUSTAL, 1968) ont signalé (en conditions sèches) la présence de racines de blé d'hiver jusqu'à $4 \mathrm{~m}$ de profondeur.

En l'absence d'argument décisif qui permettrait de choisir entre ces 2 hypothèses, nous admettrons que les 2 phénomènes peuvent exister et contribuer diversement aux remontées d'eau que l'on constate globalement.

Pour ce qui concerne les modalités du prélèvement de l'eau du sol par les cultures, le blé manifeste une aptitude particulière à dessécher intensément les horizons supérieurs alors que la luzerne développe une zone d'activité plus profonde. Ces comportements peuvent apparaître comme complémentaires et doivent vraisemblablement être considérés comme un des facteurs favorables que l'on réunit dans la pratique d'associations luzerne-graminée (BALDY, 1963).

Enfin, si l'on peut dire qu'un des buts principaux de l'étude - la détermination des flux profonds - a effectivement été atteint, il est clair que des informations plus précises sur ces flux ne pourront être obtenues qu'en complétant le dispositif expérimental par quelques piézomètres susceptibles de nous renseigner sur l'apparition d'une nappe temporaire, mais surtout par des observations continues des gradients tensiométriques en profondeur. Celles-ci permettraient en effet de déterminer à tout instant le sens et l'intensité des flux capillaires. On pourrait alors envisager une séparation plus exacte des 3 phénomènes qui concourent au «terme inconnu » du bilan hydrique : le ruissellement, l'ensemble drainage ou remontées capillaires, l'absorption racinaire profonde. Un certain nombre de difficultés nous ont jusqu'à présent empêchés de réaliser cette mesure de façon continue.

Reçu le 22 août 1983 Accepté le 27 avril 1984.

\section{RÉFÉRENCES BIBLIOGRAPHIQUES}

Baldy Ch., 1963. Cultures associées et productivité de l'eau. Ann. Agron., 14 (4), 489-534.

Baldy Ch., 1973. Progrès récents concernant l'étude du système racinaire du blé (Triticum sp.). Ann. Agron., 24 (2), 241-276.

Carvallo H. O., Cassel D. K., Bauer A., 1975. Water losses from an irrigated soybean field by deep percolation and evapotranspiration. Water Resour. Res., 2 (2), 267.

Couchat P., 1974. Mesure neutronique de l'humidité des sols. Thèse

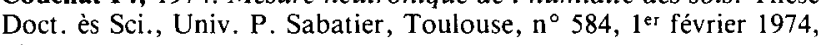
$123 \mathrm{p}$.
Daudet F. A., Valancogne C., 1976. Mesure des flux profonds de drainage et de remontées capillaires. Leur importance dans le bilan hydrique. Ann. Agron., 27 (2), 165-182.

Gardner W. R., 1958. Some steady state solutions of the unsaturated moisture flow equation with application to evaporation from a water table. Soil Sci., 85, 228-232.

Grant D. R., 1975. Comparison of evaporation measurements using different methods. Q. J. R. Meteorol. Soc., 101, 543-550.

Grebet Ph., 1965. Evapotranspiromètre pesable par dynamomètres électroniques. C. R. Acad. Agric. Fr., 51 (15), 1026-1033. 
Hillel D., 1974. L'eau et le sol. Principe et processus physiques. Vander éd. Bruxelles, $288 \mathrm{p}$.

Holmes J. W., 1950. Calibration and field use of the neutron scattering method of measuring soil water content. Aust. J. Appl. Sci., 7 , $45-58$.

Itier B., 1981. Une méthode simple pour la mesure de l'évapotranspiration réelle à l'échelle de la parcelle. Agronomie, 1 (10), 869-876.

Jongerius A., 1954. Exemple d'étude pédologique à grande échelle. Les sols du domaine de La Minière. Ann. Agron., (6), 961-981.

Katerji N. B., 1977. Contribution à l'étude de l'évapotranspiration réelle du blé tendre d'hiver. Application à la résistance du couvert en relation avec certains facteurs du milieu. Thèse Doct. Ing., Univ. Paris VII, 120 p. + fig.

Katerji N. B., Gosse G., Perrier A., Daudet F. A., 1977. Etude suivie de l'évapotranspiration réelle d'un couvert de blé et de maïs au moyen d'un dispositif automatique B.E.A.R.N. La Météorologie, VI ${ }^{\mathrm{e}}$ série, 11, 47-53.

La Rue M. E., Nielsen D. R., Hagan R. M., 1968. Soil water flux below a ryegrass root zone. Agron. J., 60, 625-629.

Lemon E. R., 1968. The measurement of height distribution of plant community activity using the energy and momentum balance approaches. In : « Fonctionnement des écosystèmes terrestres au niveau de la production primaire ». Colloque UNESCO, Copenhague 1965, 381-389.

Lipps R. C., Fox R. L., 1964. Root activity of sub-irrigated alfalfa as related to soil moisture, temperature and oxygen supply. Soil Sci., 97 (1), 4 .

Maertens C., Cabelguenne M., 1974. Intensité et limite du dessèchement du sol en relation avec l'enracinement de quelques espèces végétales. C. R. Acad. Sci., série D, 279, 2039-2043.

Nielsen D. R., Biggar J. W., Erh K. T., 1973. Spatial variability of field measured soil water properties. Hilgardia, 42, 215-260.

Nixon P. R., Lawless G. P., 1960. Translocation of moisture time in insaturated soil profiles. J. Geophys. Res., 65, 655-661.

Perrier A., Archer P., Blanco de Pablos A., 1974. Etude de l'évapotranspiration réelle et maximale de diverses cultures : dispositif et mesures. Ann. Agron., 25 (5), 697-731.

Perrier A., Itier B., Bertolini J. M., Blanco de Pablos A., 1975. Mesure automatique du bilan d'énergie d'une culture. Exemple d'application. Ann. Agron., 26, 19-40.
Perrier A., Itier B., Bertolini J. M., Katerji N., 1976. A new device for continuous recording of the energy balance of natural surface. Agric. Meteorol., 16, 71-84.

Philip J. R., 1957. Numerical solution of equations of the diffusion type with diffusivity concentration-dependent. Aust. J. Phys., 10, 2942.

Robins J. S., Pruitt O. W., Gardner W. R., 1954. Unsaturated flow of water in field soils and its effect on soil moisture investigation. Soil Sci. Soc. Am. Proc., 18, 344-347.

Rose C. W., Stern W. R., 1965. The drainage component of the water balance equation. Aust. J. Soil Res., 3, 95-100.

Slatyer R. O. , Mc Ilroy J. C., 1961. Evaporation and the principle of its measurement. In : "Practical microclimatology", UNESCO Paris éd., 909 p. + annexes, chapitre 3.

Vachaud G., Chaabouni Z., El Amani S., Vauclin M., 1983. Méthodologie d'étude du bilan hydrique d'une culture à l'échelle de la parcelle, 11 p. Comm. Colloque international sur l'emploi des techniques des isotopes et des rayonnements dans les études sur la physique des sols et l'irrigation. Aix-en-Provence, 1822 avril 1983, en cours d'impression.

Valancogne C., Daudet F., 1977. Etude in situ du potentiel hydrique sous une culture de maïs à l'aide d'un système automatique de mesure à micropsychromètres à effet Peltier. Ann. Agron., 28 (2), 137-157.

Valancogne C., Fouère A., Aho N., 1977. Contrôle de l'étalonnage basé sur l'étude neutronique d'échantillons de sol. Bull. Gr. Fr. Humidimétrie neutronique, 1, 61-65.

Van Bavel C. H. M., Stirck G. B., Brust K. J., 1968a. Hydraulic properties of a clay loam soil and the field measurement of water uptake by roots. I. Interpretation of water content and pressure profiles. Soil Sci. Soc. Am. Proc., 32, 310-317.

Van Bavel C. H. M., Brust K. J., Stirck G. B., 1968b. Hydraulic properties of a clay loam soil and the field measurements of water uptake by roots. II. The water balance of the root zone. Soil Sci. Soc. Am. Proc., 32, 317-321.

Vauclin M., Haverkamp R., Vachaud G., 1982. Analyse de variance sur la teneur en eau mesurée à l'humidimètre neutronique. Bull. Gr. Fr. Humidimétrie neutronique, 12, 17.

Wilcox J. C., 1960. Rate of soil drainage following an irrigation. II. Effects on determination of rate of consumptive use. Can. J. Soil Sci., 40, 15-27. 